\title{
In-Vitro Potential of Crude Extracts of Selected Garden Herbs for Mastitis Management in Zambia
}

\author{
Ngwisha Joshua ${ }^{1,7 a}$, Samutela Tillika Mulemba ${ }^{1,2}$, Phiri Bruno ${ }^{1,8}$, Zulu Mbawe ${ }^{3}$, Mwasinga \\ Wizaso $^{6}$, M N Balakrishnan Nair ${ }^{4}$, Choongo Kennedy ${ }^{5 a}$ and Hangombe Bernard Mudenda ${ }^{1 a}$
}

'Department of Paraclinical Studies, School of Veterinary Medicine, University of Zambia, Lusaka, Zambia

${ }^{2}$ Department of Biomedical Sciences, School of Health Sciences, University of Zambia, Lusaka, Zambia

${ }^{3}$ Department of Basic Sciences, Michael Chilufya Sata School of Medicine, Copperbelt University, Ndola, Zambia

${ }^{4}$ University of Trans-Disciplinary Health sciences and technology, Bengaluru, India

${ }^{5}$ Department of Biomedical Sciences, School of Veterinary Medicine, University of Zambia, Lusaka, Zambia

${ }^{6}$ Department of Disease control, School of Veterinary Medicine, University of Zambia, Lusaka, Zambia

${ }^{7}$ Zambia Air Force, Mumbwa Base, Zambia

${ }^{8}$ Central Veterinary Research Institute, Lusaka, Zambia

${ }^{a}$ The authors contributed equally to the work

Corresponding author: Joshua Ngwisha, Mailing Address: Zambia Air Force Mumbwa Station Sick

Quarters. P.O Box 830113, Mumbwa, Zambia. Tel: +260971232887, Email: drjoshuangwisha@gmail.com

DOI: https://doi.org/10.53974/unza.jabs.5.1.523

\section{Abstract}

Introduction: The advent of bovine mastitis associated antimicrobial resistance has necessitated development of alternative herbal remedies. In this study, the researchers tested, invitro, the antimicrobial efficacy of three crude extracts [1] aqueous, [2] ethanol and [3] ethyl acetate extracts of Zambian cultivated Aloe barbadensis Miller (Aloe vera) and Curcuma longa (Turmeric) and made comparisons to synthetic anti-mastitis formulations.

Methods: The active compounds of these herbs were obtained as crude extracts using the solvents water, ethanol and ethyl acetate. The test microbes comprised of ten recognized controls including Escherichia coli and Pseudomonas aeruginosa and eightyfour field isolates (all gram positive bacteria) from clinical and subclinical mastitis milk samples from selected districts of the Southern province of Zambia belonging to the genera Staphylococcus, Streptococcus, Bacillus and Lactobacillus. The agar dilution method was used to determine the antimicrobial activity by observing the susceptibility, which was graded, as susceptible, intermediate or resistant. Commercial synthetic anti-mastitis preparations served as positive controls and their susceptibility was compared to that of the herbal preparations. The Minimum Inhibitory Concentrations (MIC) of the herbal formulation was determined using two-fold serial dilutions.

Results: On average, the agar dilution trials revealed; 41.8 per cent resistance, 13 per cent intermediate and 45.3 per cent susceptibility for the herbal formulation while the synthetic antimicrobials produced 23.5 per cent resistance, 5.2 per cent intermediate and 71.3 per cent susceptibility. The gram negative controls tested were generally resistant.. Mean MIC was $11.5 \mathrm{mg} / \mathrm{ml}$ at the 5 per cent serial dilution for the herbal extract compared to the Cephalexin (semi-synthetic 
antimicrobial) MIC for $S$. aureus at $0.5 \mathrm{mg} / \mathrm{L}$ or $0.0005 \mathrm{mg} / \mathrm{ml}$.

Conclusion: The herbs Aloe vera and Curcuma longa from Zambia showed antimicrobial efficacy on bovine mastitis causing microbes at 5 per cent MIC and could prospectively be assessed as an economical alternative to synthetic anti-mastitis preparations as well as an effective adjunct in fighting antimicrobial resistance to the benefit of the most affected and resource limited small-scale dairy farmers.

Keywords: Aloe vera, curcuma longa, mastitis, ethno veterinary medicine, agar dilution

\section{Introduction}

Mastitis refers to udder inflammation. Bovine mastitis is common in both developed and developing countries, and is a significant disease in the dairy industry worldwide. Bacteria are the most implicated causes of mastitis, and gram-positive bacteria, such as Staphylococcus are among the most common udder pathogens in Zambia [1]. Mastitis causes direct and indirect losses through decrease in milk production, discarded or rejected milk and costs of disease treatment and management including death of cows through culling [2].

Mastitis is usually treated with antimicrobials through intra mammary infusions. However, the use of these antimicrobials such as antibiotics has been associated with antimicrobial resistance [3]. This situation has made the search for alternative treatment methods necessary [4].

Herbs are proving to be an excellent natural resource used as an alternative therapy for mastitis [5]. Herbal formulations for the treatment of mastitis have shown to have no residual effects in the milk and blood of the treated cows subsequent to extra-mammary application, presumably due to their organic nature that enables ease of metabolism [6]. Additionally, researchers have noted the availability and widespread use of Ethno-Veterinary Medicine (traditional animal health care) in rural parts of Southern Africa, and have emphasised the need to undertake further research into the potential and validation of ethno-veterinary medicines in view of reducing the use of antibiotics in infectious diseases and discovering alternative treatments to curb antimicrobial resistance $[7,8]$.

The diverse vegetation of Zambia, along with cultivation of certain highly used medicinal plants, could upscale ethno-veterinary science and practice in Zambia. However, research into the effectiveness of these herbs as Veterinary Medicine and their relevance to providing alternative, local solutions to battle against antimicrobial resistance in Zambia is meagre.

This study aimed at assessing the in-vitro antimicrobial activity of an Aloe vera and Curcuma longa formulation on microbes that cause mastitis. It provided investigational indications on the use of locally available herbs in Zambia, for the management of livestock diseases, and also carried the potential of providing some solutions to antimicrobial resistance in Zambia.

\section{Materials and Methods Study Design}

The study was experimental in nature and intended to establish the 
antimicrobial activity of extracts of Zambian cultivated Aloe vera and Curcuma longa on bovine mastitis causing micro-organisms in-vitro. Corroborated in-vitro methods were used to screen the combined formulated crude extract of $A$. vera and $C$. longa for antimicrobial activity. The effectiveness was compared to the locally available synthetic antimicrobials for treatment of mastitis.

Five trials were conducted to determine the antimicrobial activity of the combined formulation extracts, and the findings were compared for each of the trials to the commercially available synthetic antimicrobials used for mastitis management in Zambia. This served as a probable indicator of the in vivo efficacy of the extracts against microbes implicated in bovine udder infection.

\section{Plant Materials}

Whole plant materials, A. vera and $C$. longa used for this study were procured from Luanshya and Lusaka districts of Zambia (See Figure 1 below) following a non-participatory rapid appraisal [9]. Three hundred grams of $A$. vera leaves and 60 grams C. longa rhizomes were dried in a drying oven at $35^{\circ} \mathrm{C}$ for seven days as described by Punnaimurthy [10].

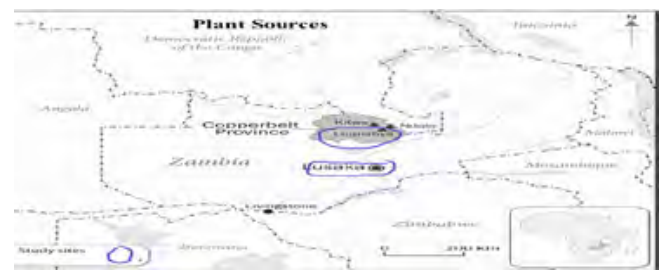

Figure 1: Map showing plant source towns (blue circle) in Zambia, Africa. (Source: Google maps)

\section{Preparation of Herbal Extracts}

After drying as referred to above, the herbal extracts were macerated in the solvents aqueous, ethanol and ethyl acetate as described by Punniamurthy and Ramakrishnan (2015) [11]. Thereafter, $1 \mathrm{ml}$ of 100 per cent Dimethylsulfoxide (DMSO) was added to the extracts for complete dissolution of all solid extract particles.

\section{Bacteria used for the Determination of Antibacterial Activity}

The Clinical and Laboratory Standards Institute (CLSI) antimicrobial efficacy screening recommended gram positive Staphylococcus aureus (ATCC 25923) and gram negative Escherichia coli as well as Pseudomonas aeruginosa were used in the first two preliminary trials [12]. The pure isolates were pooled from the field isolates of the University of Zambia, School of Veterinary Medicine (UNZA-SoVM). In the third trial, Streptococcus, Bacillus and Candida isolates were used and tested accordingly.

In the fourth and fifth trials, critical case isolates derived from clinical and subclinical mastitis milk samples for cows from Dairy Cooperatives in Mazabuka, Monze, Kalomo, Namwala and Choma districts of Zambia were aseptically collected and transported under ice in a cooler box to the laboratory.

The laboratory method for isolation and detection of microbes from the milk samples, with the only modification being the non-addition of the 6 per cent Sodium Chloride $(\mathrm{NaCl})$ for the enrichment which is $S$. aureus specific was as described by Phiri [13] and Teanhagen et al [14]. The field cultures were identified by gram staining and 
biochemical reactions as described by Bisen et al [15]. Following phenotypic confirmation of the isolates, the culture colonies were then constituted to the $0.5 \mathrm{McF}$ arland and processed for the determination of antimicrobial activity.

\section{Culture Media and Antibiotics}

Mueller-Hinton Agar (MHA) media (Oxoid, United Kingdom) was used for the growth of bacterial cultures as well as for the antibacterial determination tests, while Mueller-Hinton broth (Oxoid, UK) was incorporated for MIC determination. Brain Heart Infusion and Blood agar (Oxoid, UK) were also utilised for enrichment and culture of the fastidious bacteria respectively.

Common Zambian market available anti-mastitis preparations Terrexin ${ }^{\circledR}$ (Each injector of 10 gram contains: Cefalexin $200 \mathrm{mg}$ and Kanamycin monosulfate 100,000 IU) and Rilexine 200 L.C ${ }^{\circledR}$ (containing Cefalexin 100mg, Neomycin Sulphate $100 \mathrm{mg}$ and Prednisolone $10 \mathrm{mg}$, per $10 \mathrm{ml}$ ) were utilised for comparative purposes and as experimental controls.

\section{Determination of Antimicrobial Activity}

Antimicrobial activity of all the extracts, was tested by the agar dilution method. The initial extract volume $\left(\mathrm{V}_{\mathrm{i}}\right)$ was diluted $(0.5 \mathrm{ml}$ extract into $19.5 \mathrm{ml}$ agar for initial trials and $1 \mathrm{ml}$ into $19 \mathrm{ml}$ for latter trials) into the media to make up to $20 \mathrm{ml}$ (final volume $\left(\mathrm{V}_{\mathrm{f}}\right)$ ) before agar solidification at temperatures of between $45-55^{\circ} \mathrm{C}$ and petri-dish plated. After solidification and drying, the test saline dissolved bacterial isolates were spot inoculated onto the MHA at the CLSI recommended inoculum rate of $0.5 \mathrm{McF}$ arland and marked for bacterial test strain identification after which the plates were incubated at $37^{\circ} \mathrm{C}$ for twenty four hours. In similar manner, plates with dilute antibiotic (Terrexin ${ }^{\circledR}$ and Rilexine $^{\circledR} ; 0.5 \mathrm{ml}$ antibiotic to $19.5 \mathrm{ml}$ agar) and undiluted agar as well as DMSO controls were also prepared and spot inoculated with the test bacterial strains for comparative purposes.

Observations were recorded based on the CLSI recommended susceptibility analysis and graded as Susceptible (S), Intermediate (I) or Resistant (R). The bacterial cultures were observed 24 hours post incubation and observation was continued where susceptibility was evident, over a period ranging from ten to thirty-seven days in order to try and understand if the effects were bacteriostatic or bactericidal and the susceptibility observations were recorded.

\section{Minimum Inhibitory Concentration (MIC) Determination}

To determine the MIC, serial dilutions in Mueller-Hinton Broth were prepared beginning with, a 10 per cent extract in agar initial dilution to least final dilution concentrations of 1.25 per cent and 0.15625 per cent for the first and second trials respectively. Staphylococcus aureus was utilised for the MIC determination of which a $100 \mu 1$ stock control was added to all the dilutions individually, vortexed and incubated for twenty-four hours, and then inoculated for each serial dilution onto Nutrient agar and thereafter, observations were made and recorded. Ethanol extracts were used for the MIC determination. The recordings were related to the MIC values for the synthetic antimicrobials.

\section{Ethical Considerations}

Ethical approval was obtained from the University of Zambia Biomedical 
Research Ethics Committee (UNZABREC), approval number 10732020. Permission to collect critical case milk samples was sought from the farmers. All severely inflamed cows were treated on site, and susceptibility results were communicated promptly.

\section{Results and Discussion}

Determination of Antimicrobial activity of the $A$. vera and $C$. longa formulation The findings from the agar dilution of the herbal formulation extracts associated to the synthetic preparations for the five trials are highlighted and discoursed below.

\section{Trial 1}

In the first trial gram negative $E$. coli and $P$. aeruginosa were resistant and accounted for the most part of the 48 per cent resistance recorded for the herbal formulations cumulatively (Figure 3a). The Staphylococcus aureus control strain (ATCC 25923) and S. aureus isolates were the most susceptible to the herbal treatment with alternations noted between intermediate susceptibility and susceptibility over the thirty-seven days observation period for this trial, indicative of bacteriostatic properties. Overall, the bacteria were susceptible to the treatment (Figure 3a), on 37 per cent of all observations and when combined with the 15 per cent intermediate susceptibility, yielded a total of 52 per cent susceptibility on all observations made. When compared to the susceptibility results of the synthetic antimicrobials $\left(\right.$ Terrexin $^{\circledR}$ and Rilexine ${ }^{\circledR}$, Figure 3b) in trial 1, it was noted that the gram negative $E$. coli and $P$. aeruginosa were again the most resistant and accounted for over two thirds of the 49 per cent resistance recorded for the anti- mastitis preparations cumulatively. The $S$. aureus also showed some level of resistance with time to the synthetic antimicrobials during the thirty-seven day period of observation. The $S$. aureus isolated from mastitis cases was the most susceptible to this treatment with alternations noted between intermediate susceptibility and susceptibility. In this trial, the bacteria (E. coli, P. aeruginosa and $S$. aureus) was susceptible to the synthetic antimicrobial treatment on 44 per cent of all observations and in combination with the 7 per cent intermediate susceptibility yielded a total of 51 per cent susceptibility from all observations recorded.

The overall bacterial susceptibility to the herbal formulation (Figure 3a) in trial 1 was 52 per cent (intermediate plus susceptible) compared to 51 per cent for the synthetics (Figure 3b). The herbal formulation; therefore, performed only a percentile better than the synthetics during this preliminary trial. In an agar well diffusion study, in India in 2015, Punniamurthy and Ramakrishnan reported that the A.vera and C. longa formulation at $200 \mu \mathrm{L}$ per well, showed inhibitory effects against $E$. coli and $S$. aureus under in-vitro conditions [16]. Our agar dilution test findings at concentrations of 2.5-10 per cent however showed that E. coli was resistant but comparably susceptible on the $S$. aureus. This difference could be attributable to the method and higher dosage used. 


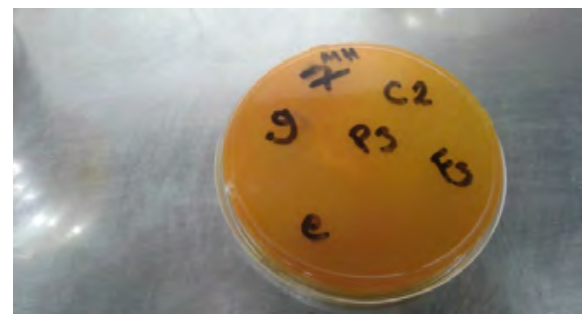

Figure 2a

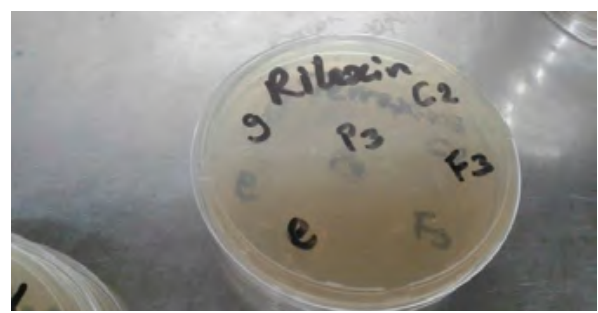

Figure $2 b$

Figure $2 \mathrm{a}$ and $2 \mathrm{~b}$ : Spot inoculated plates showing $A$. vera and $C$. longa formulation (a) diluted in MHA versus Synthetic Antibiotic/ positive control (b) diluted in MHA.

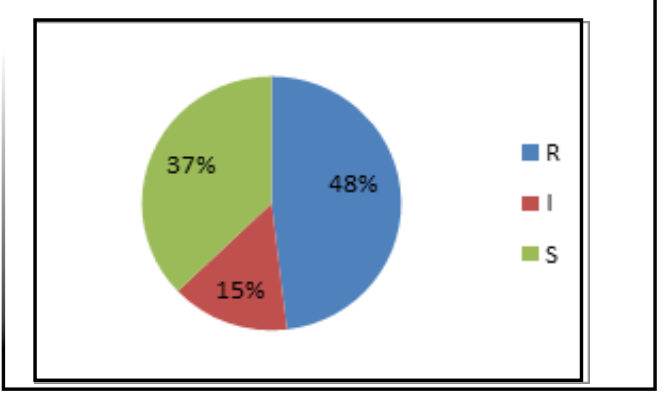

Figure 3 a $(n=210)$

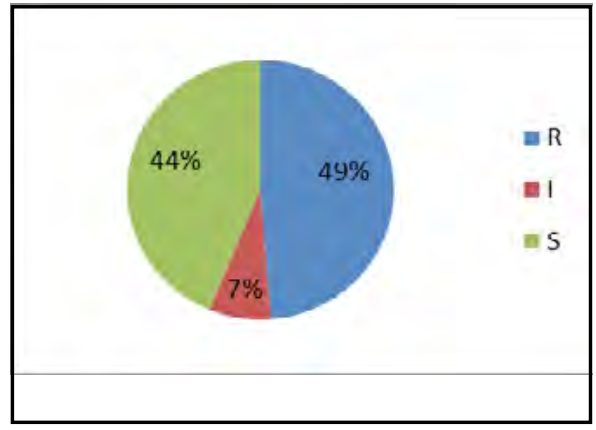

Figure $3 \mathrm{~b}(\mathrm{n}=80)$

Figure 3(a and b): Trial 1 (a) A. vera and $C$. longa formulation versus (figure 3b) Synthetic Anti-mastitis preparations activity on all isolates ( $\mathrm{R}$ is resistant, I intermediate and $\mathrm{S}$ is susceptible).

\section{Trial 2}

In the second trial, results of the herbal formulation (Figure $4 a$ ) revealed that the gram negative isolates $(E$. coli and $P$. aeruginosa) were again consistently resistant and accounted for over three quarters of the 72 per cent resistance recorded cumulatively. The Staphylococcus aureus, control strain and the field mastitis isolate accounted for less than 10 per cent of the overall resistance. Overall, the bacteria was susceptible to the formulation on 8 per cent of all observations and in combination with the 20 per cent intermediate susceptibility yielded a total of 28 per cent susceptibility from all observations made as graphically represented in Figure 4a.

However, when analysed separately, results from the gram positive isolates (Figure 4b), exhibited 26 per cent susceptibility, and when combined with the 59 per cent intermediate susceptibility equalled 85 per cent susceptibility.

The intermediate susceptibility was the most predominant in the course of this trial for the herbal formulations. The isolates, in the second trial, were for the first time tested at three times the dosage of some select extracts used in the first trial. As graphically summarised in Figure 5, the findings revealed that the gram negative isolates $E$. coli and P. aeruginosa were again resistant and accounted for the whole of the 66 per cent resistance recorded cumulatively. On the whole, the bacteria was susceptible to this higher dose (Figure 5a) on 22 per cent of all observations and in combination 
with the 12 per cent intermediate susceptibility yielded a total of 34 per cent susceptibility from all observations made in this phase of the second trial.

However, when analysed in isolation, the gram positive isolates (Figure $5 \mathrm{~b}$ ) exhibited 65 per cent susceptibility, and when combined with the 35 per cent intermediate susceptibility, equalled 100 per cent susceptibility at this higher application.

This study suggested for the first time that increasing the application dose of the formulation may imply an increase in efficacy. The limited scope of our study could not enable probing further into any higher dose toxicity effects, but this may not be absolutely necessary as the formulation has been proven to be safe and effective by Balakrishnan and colleagues at the current formulation proportions [17].

In keeping with the performance of the synthetics in the second trial (Figure 6), the E. coli was found to be the sole contributor to the 7 per cent resistance noted cumulatively. In this trial, the bacteria were susceptible to the synthetics on 85 per cent of all observations and in combination with the 8 per cent intermediate susceptibility yielded a total of 93 per cent susceptibility from all observations made.

The synthetic antimicrobials (Figure 6) performed better than the herbal formulations (Figure $4 \mathrm{a}$ ) in trial two. The poor performance of the herbs could be attributed to the contemptible drying conditions of the herbs during the processing stages for this trial. Consequently, this limitation in our drying conditions as reviewed by Rocha et al., can be held to be of paramount importance in the extraction process [18]. Rocha et al., in their review of the influence of the drying process on the quality of medicinal plants concluded that the drying method, velocity and temperature of drying air influence the quantity and quality of the active ingredients present in aromatic and medicinal plants [19]. However, justification of this postulation was beyond the scope of our study.

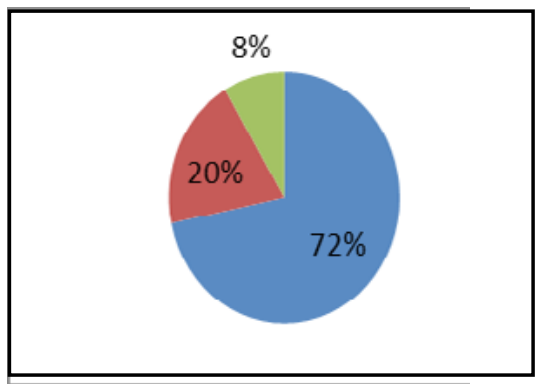

(a) $(n=732)$

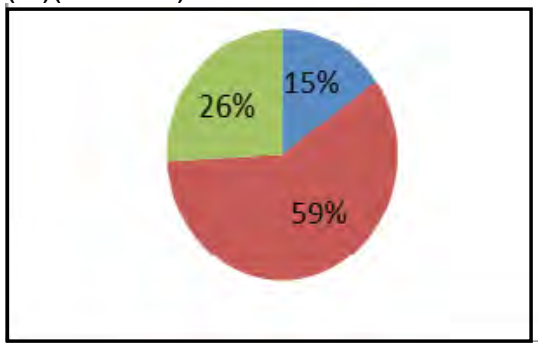

(b) $(n=244)$

Figure 4 (a)-(b): Trial 2 (a) A. vera and $C$. long a formulation preparations' activity on; all isolates and (b) on gram positive isolates only ( $\mathrm{R}$ is resistant, I intermediate and $\mathrm{S}$ is susceptible).

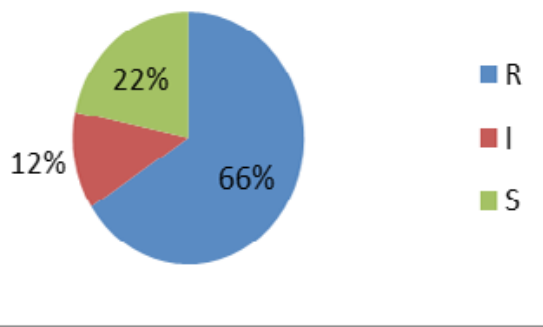

(a) $(\mathrm{n}=732)$ 


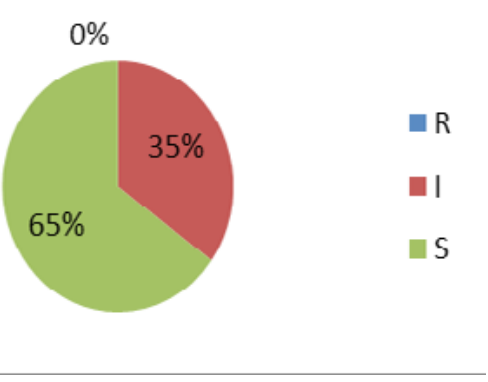

(b) $(\mathrm{n}=40)$

Figure 5 (a)-(b): Trial 2 Select Triple yield Combined $A$. vera and $C$. longa formulation susceptibility on; (a) all isolates and (b) on gram positive isolates only ( $\mathrm{R}$ is resistant, I intermediate and $\mathrm{S}$ is susceptible).

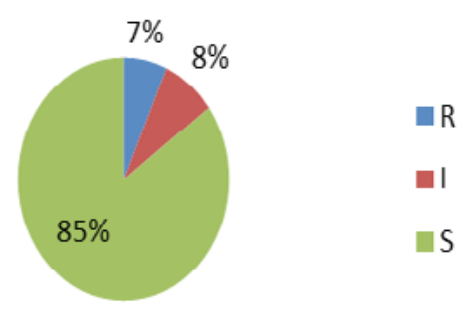

Figure 6: Trial two Synthetic antimastitis preparations susceptibility ( $\mathrm{R}$ is resistant, I intermediate and $\mathrm{S}$ is susceptible, $\mathrm{n}=60$ ).

\section{Trial 3}

In the third trial, when the herbal formulation was assayed (Figure 7), all the microbes tested (Bacillus babosus, Candida albicans and Streptococcus) exhibited varying degrees of resistance, which was cumulatively recorded at 40 per cent. Bacillus babosus, was the most susceptible (Figure 7a), while Streptococcus displayed equality between susceptibility and resistance based on the observations made. The Candida albicans was noted to be more susceptible than resistant to the herbal formulation.
The microbes during trial three (Figure $7 b$ ) were susceptible to the formulation on 54 per cent of all observations and in combination with the 6 per cent intermediate susceptibility yielded a total of 60 per cent susceptibility.

The synthetic antibiotics in trial 3 had no inhibitory effect on the Candida albicans (fungi) as shown in Figure 8(a) which in itself, accounted for two thirds of the overall resistance on all observations. Streptococcus isolates were the most susceptible to the synthetic antimicrobials while Bacillus babosus showed the least susceptibility of the three pathogenic microbial isolates. Statistically, the combined susceptibility (Figure 8b) of the microbes to the synthetic antimicrobials was 62 per cent (56\% Susceptible and $6 \%$ Intermediate) from all observations.

Therefore, the synthetic antimicrobial (Figure 8b) was 2 per cent superior over the herbal formulation (Figure $7 b$ ) in the third trial despite not being able to suppress the growth of the C. albicans. It should, however, be noted that the commercial anti-mastitis formulations are a mixture of both gram-positive and gram-negative antimicrobials but have no antifungal. The herbal extract, on the other hand, displayed anti-gram positive and antifungal activity.

The results from the third trial are similar to the findings of Alemdar and Agaoglu, who in their investigation revealed that Aloe vera juice had antimicrobial activity against microbes such as Candida albicans and Bacillus sphericus [20]. Therefore, the antifungal activity of the formulation on the $C$. albicans could be attributed to the Aloe, implying that the formulation could be advantageous for the management of mastitis of fungal aetiology. Epidemiologically, bacteria are the most 
prevalent causes of mastitis, however, as shown by Langdon and Ben-Ami et al., prolonged use of antimicrobials suppress the commensal bacteria, which leads to increased prevalence of secondary fungal infections $(21,22)$. The resultant as described by Soll, is a polymicrobial disease of mixed mycoses with secondary opportunistic bacteria, which results into higher antimicrobial use [23]. Despite our narrow study scope, our findings and those of Alemdar and Agaoglu highlighted above, indicate that the herbal formulation may provide synergism in management of persistent mastitis in which opportunistic fungal infections may cause complications.

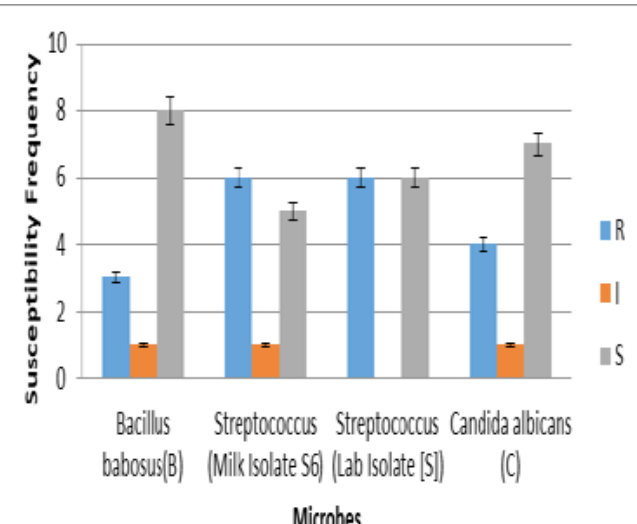

(a)

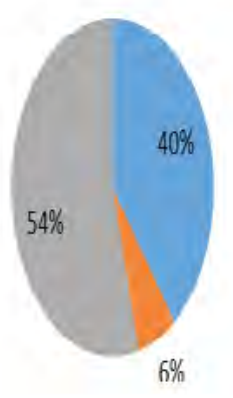

(b)

Figure 7 (a) and (b): Trial 3 Herbal preparations' activity on; (a) individual isolates and (b) cumulatively on all isolates ( $\mathrm{R}$ is resistant, I intermediate and $S$ is susceptible, $n=48$ ).
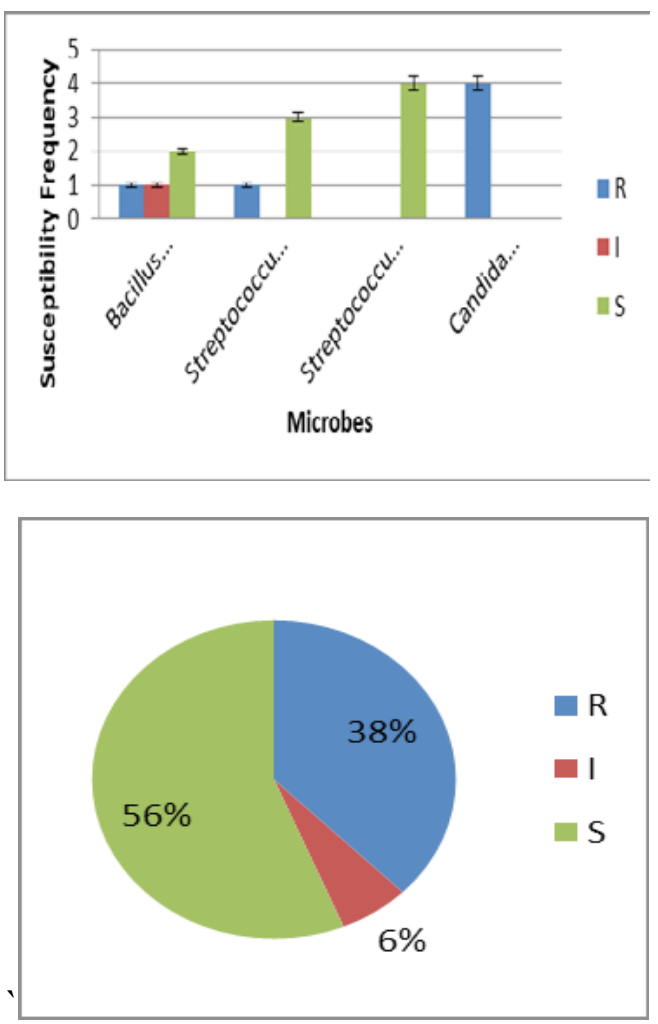

Figure 8 (a) \& (b): Trial 3 Synthetic Antibiotics antimicrobial activity on (a) individual isolates and (b) on all isolates cumulatively ( $\mathrm{R}$ is resistant, I intermediate and $\mathrm{S}$ is susceptible, $\mathrm{n}=16$ ).

\section{Trial 4}

The fourth trial involved collection of twenty-six [26] critical case mastitis samples. The agar well diffusion method was experimented with for this trial and the zones of inhibition recorded for the three treatments had their variances analysed (ANOVA at 
$\alpha=0.05$ ) using Microsoft Excel 2010 with the hypothesis tested for the very first time, that there was no significant difference in the means of the three treatments (herbal formulation, Rilexine ${ }^{\circledR}$ and Terrexin $\left.{ }^{\circledR}\right)$. This hypothesis after analysis was rejected as the $\mathrm{F}$ (observed) was greater than the $\mathrm{F}$ (critical). Therefore, this entails that the mean zones of inhibition for the synthetic antimicrobials $\left(\right.$ Rilexine ${ }^{R}=$ $1.09 \mathrm{~cm}$, Terrexin $\AA=1.07 \mathrm{~cm})$ were more significant and superior to the mean of the herbal formulation (mean= $0.48 \mathrm{~cm}$ ).

The presence of only gram positive isolates (Staphylococcus, Streptococcus, Streptococcus pneumoniae, Lactobacillus and bacillus spores) from the mastitis critical case samples during trial four favours use of the herbal formulation, which in all trials, proved to be efficacious against gram positive bacteria. The revelation of such isolates reaches agreement with the findings of Eriksson, who reported that the most common udder pathogens in Mapepe, Batoka and Choma areas of Zambia were the gram positives [24].The high efficacy of the herbal formulation on the gram positives in line with this known problem in Zambia as noted by Erikson and this study, necessitates further scientific justification for the adoption of alternative medicines such as this herbal formulation in Zambia. The knowledge gap in the use of agar diffusion for comparison of herbs to conventional treatments for mastitis treatment, restricted the referencing for our study.

\section{Trial 5}

In the fifth trial, the cultured microbes depicted high levels of susceptibility to the herbal formulation (Figure 9). On the overall, 82 per cent of the sample isolates were susceptible to the herbal formulation and when combined with 11 per cent intermediate susceptibility, totalled 93 per cent susceptibility, leaving only 7 per cent resistance for this trial.

The isolates in the fifth trial were 100 per cent susceptible to the synthetic antimicrobials, and, therefore, marginally (7\%) superior to the crude herbal extracts $(93 \%$; $\mathrm{S}+\mathrm{I})$.

The high susceptibility findings in trial five could be attributed to the use of freshly prepared extracts, as the extracts in this trial were immediately diluted into the agar at the end of the extraction process unlike in previous trials where the isolates had been stored at $-20^{\circ} \mathrm{C}$ for periods of between one day to three weeks prior to use. This study, therefore, agrees with the inferences of the year 2013 evaluation by Laher et al., which showed that the fresh samples of the majority of plant extracts indicated better antibacterial and antifungal activities in comparison to the stored samples

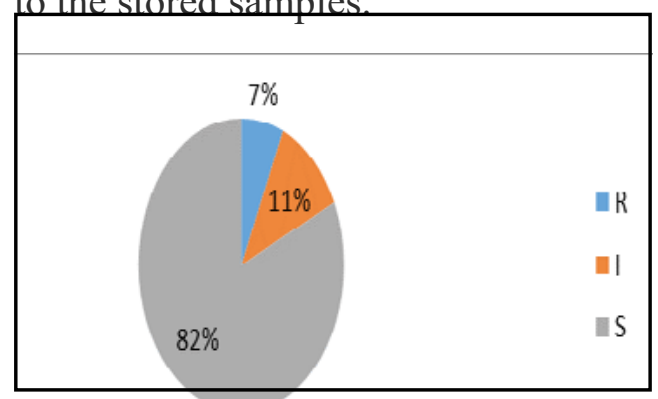

Figure 9: Trial 5 Herbal Extracts Susceptibility on Field Mastitis Isolates ( $\mathrm{R}$ is resistant, I intermediate and $\mathrm{S}$ is susceptible, $n=150$ ) When averaged for all the agar dilution trials, this previously undocumented comparison, revealed; 41.8 per cent resistance, 13 per cent intermediate susceptibility 
and 45.3 per cent susceptibility for the herbal formulation while the synthetic antimicrobials produced 23.5 per cent resistance, 5.2 per cent intermediate and 71.3 per cent susceptibility. The absence of in-vitro reference points for the comparison of this A. vera and $C$. longa formulation to synthetic anti-mastitis preparations is not only a limitation for our study, but can also be viewed as an opportunity to fill this knowledge gap.

\section{Determination of Minimum Inhibitory Concentration (MIC)}

Three ethanol extracts were purposively selected for MIC determination. From the three categorically analysed herbal ethanol extracts for the MIC determination on Staphylococcus aureus, the 5 per cent dilution was inferred as the MIC, and the average concentration arising from the initial extract concentrations $(\mathrm{m} / \mathrm{v})$ was determined to be $11.5 \mathrm{mg} /$ $\mathrm{ml}$. This is the mean arising from the $13 \mathrm{mg} / \mathrm{ml}$ for the first sample (9T2) in run 1 and from run 2, the correlated concentrations at 5 per cent dilution which were $12.5 \mathrm{mg} / \mathrm{ml} \& 9 \mathrm{mg} / \mathrm{ml}$ for extract 7(T1) and 18(T2) respectively. These findings vary slightly from those of Punniamurthy, who found an ethanol extract MIC on $S$. aureus of $20 \mathrm{mg} / \mathrm{ml}$. This noted variation could hypothetically be attributable to several factors including concentration of the inoculant in addition to potential human error. Since Cephalexin MIC as determined in 2001, by Andrews on $S$. aureus is much lower at $0.5 \mathrm{mg} / \mathrm{L}$, it can be implied, that comparison of the herbal preparation MIC to that of the synthetic anti-mastitis preparations is uneven [27]. This could be probably attributable to the wide variation of the active constituents which are more purified for the synthetic preparations in comparison to the crude extracts. However, this speculation may require phytochemical analysis which was beyond the scope of our study

The introduction and application of ethno-veterinary practices in Zambia may give rise to a reduction in the use of antibiotics, possible elimination/ reduction of antibiotics use in animal production and animal products as well as reduce antimicrobial resistance in the long run.

\section{Conclusion}

Ethno-veterinary herbal preparations comprising of Zambian grown Aloe vera and Curcuma longa, possess gram positive antibacterial and antifungal spectrum of activity on bovine mastitis and other pathogenic microorganisms in-vitro and carry the prospect of being utilised as alternatives to locally used synthetic anti-mastitis preparations in Zambia, at an MIC of 5 per cent. This study recommends to policymakers, interest groups and other researchers, for the validation of such and other useful local herbs and formulations in Zambia to the benefit of the most affected and resource limited smallscale dairy and organic farmers. An intervention impact assessment on the reduction of antimicrobial residues in milk, coupled with mitigating the development of antimicrobial resistant pathogens associated with bovine mastitis, may also be necessary. It is further recommended to academia and policymakers to consider developing a cadre of ethnoveterinary practitioners through the establishment of a postgraduate diploma in ethno-veterinary practices in Zambia, as this may aid in natural 
resource development, utilisation and job creation.

\section{Acknowledgements}

We sincerely thank Kafakumba training centre, Mutepa Manchemba and the Mwiinga's for availing their herbs. Special thanks also go to the University of Zambia School of Veterinary Medicine laboratory scientists and technicians who were instrumental throughout the experimentation.

\section{Authors' Contributions: NJ,} $\mathrm{KC}$ and $\mathrm{BMH}$ conceived the idea, MNB provided and guided on the formulation, NJ, STM, MW, BSJP, collected the bacterial samples, $\mathrm{NJ}$, $\mathrm{BMH}$ and $\mathrm{KC}$ analysed the data, $\mathrm{NJ}$ drafted the manuscript, BSJP, MNB, STM, BMH, KC and ZM critically reviewed the manuscript. All authors read and approved the final draft of the manuscript. $\mathrm{KC}$ and $\mathrm{BMH}$ supervised the research.

\section{Competing Interests}

The authors declare that there are no conflicts of interest associated with this study.

Research Funding: This research was funded by Prof BM Hang'ombe and the Deutsche Gesellschaft fur Internationale Zusammenarbeit (GIZ) under their Green Innovation Centres (GIC) project in Zambia.

\section{References}

1. Eriksson L. Prevalence of subclinical mastitis and udder pathogens in small holder dairy farms in Mapepe, Batoka and Choma areas in Zambia, Examensarbete, 2013:64.

2. Halasa $T$, Huijps $K$, Østerås $O$, and Hogeveen $H$. Economic effects of bovine mastitis and mastitis management: A review, Veterinary Quarterly. 2007, 29:1, 18-31.

3. KundaB. 'Hygienic and compositional quality of raw milk produced by smallholder dairy farmers in Lusaka province of Zambia', Masters Dissertation, University of Zambia 2015.

4. Van't Hooft KE, Groot MJ, and Gebru G. Natural livestock Farming: Piloting a strategy to improve milk quality and reduce Anti-Microbial Resistance. Appro Poult Dairy \& Vet Sci. 2017; $1[3]$.

5. Mushtaq S, Shah AM, Shah A, Lone SA, Hussain A, Hassan QP, et al. Bovine mastitis: An appraisal of its alternative herbal cure. Microb Pathog. 2018;114:357-361.

6. Balakrishnan MN, Punniamurthy N, and Kumar SK. Ethno-Veterinary Sciences and Practices for Reducing the Use of Antimicrobial and Other Veterinary Drugs in Veterinary Practices-TDU Experience. In Sejian V, Isloor S, Rahman AS, Bhatta R, editors. PCVC7: Proceedings of the 7th Pan Commonwealth Veterinary Conference; Bengaluru, India, 3-7 March 2019.p 108.

7. Syakalima M, Simuunza M, and Zulu VC. Ethno-veterinary treatments for common cattle diseases in four districts of the Southern Province, Zambia, Veterinary World.2018; 11[2]: 141-145.

8. Chinsembu KC, Negumbo J, Likando $\mathrm{M}$, and Mbangu $\mathrm{A}$. An ethnobotanical study of medicinal plants used to treat livestock diseases in Onayena and Katima Mulilo, Namibia. South African Journal of Botany. 2014; 94 :101-107.

9. Kemm J, Parry J, and Palmer S. Health Impact Assessment, Oxford University Press, Oxford, United 
Kingdom, 2004.

10. Punniamurthy N. Ethnoveterinary herbal health care of dairy cattle. In Ranganathan V, Senthil KP and Elamaran A.(ed), Propagation of Ethnoveterinary Herbal Knowledge. Tamil Nadu, India: Shanlax Publications, 2017 pp 95-96.

11. Punniamurthy N, and Ramakrishnan N. Antimicrobial Activity of Ethno Veterinary Herbal Fresh Preparation for Mastitis. Kerala Veterinary Congress, Kerala, India, 2015, pp 398401.

12. Clinical and Laboratory Standards Institute. "Methods for dilution antimicrobial sensitivity tests for bacteria that grow aerobically; approved standard-eighth edition. 2009, M07-A8." National Committee for Clinical Laboratory Standards 29.

13. Bruno SJP. Characterisation of staphylococcus aureus associated with the dairy value chain in Western, Lusaka and Southern provinces of Zambia: Reducing the risk of virulent, enterotoxigenic and antibiotic resistant strains to consumers. $\mathrm{PhD}$ Thesis, University of Zambia, Zambia. 2021.

14. Tenhagen BA, Vossenkuhl B, Kasbohrer A, Alt K, Kraushaar B, Guerra B, et al. Methicillin-resistant Staphylococcus aureus in cattle food chains - prevalence, diversity, and antimicrobial resistance in Germany. Journal of Animal Science, 2014; 92, 2741-2751.

15. Bisen PS., Prasad GBKS. and Debnath M. 2012. Microbes: Concepts and applications. John Wiley \& Sons, Inc, USA.

16. Punniamurthy N, and Ramakrishnan N. Antimicrobial Activity of Ethno Veterinary Herbal Fresh Preparation for Mastitis. Kerala Veterinary Congress, Kerala, India, 2015, pp 398-
401.

17. Balakrishnan MN, Punniamurthy N, Mekala P, Ramakrishnan N, and Kumar SK. Ethno-veterinary Formulation for Treatment of Bovine Mastitis. Journal of Veterinary Sciences. 2017, S1, pp 25-29.

18. Rocha RP, Melo EC, and Radunz LL. Influence process on the quality of medicinal plants: A review. Journal of Medicinal Plants Research, 2011, 5(33): 7076-7084.

19. Alemdar S, and Agaoglu S. Investigation of In-vitro Antimicrobial Activity of Aloe vera juice. Journal of Animal and Veterinary Advances, 2009, 8: 99-102.

20. Langdon A, Crook N, Dantas G. The effects of antibiotics on the microbiome throughout development and alternative approaches for therapeutic modulation. Genome Med. 2016; 8[1]:39.

21. Ben-Ami R, Olshtain-Pops K, Krieger M, Oren, I, Bishara, J, et al. "Antibiotic exposure as a risk factor for fluconazole-resistant Candida bloodstream infection." Antimicrobial Agents and Chemotherapy. 2012, vol. 56,5: 2518-23.

22. Soll DR, 2002. Mixed Mycotic Infections. In: Brogden KA, Guthmiller JM, editors. Polymicrobial Diseases. Washington (DC), ASM Press; Chapter 17. Available from: https://www.ncbi.nlm.nih.gov/books/ NBK2485.

23. Eriksson L. Prevalence of subclinical mastitis and udder pathogens in small holder dairy farms in Mapepe, Batoka and Choma areas in Zambia, Examensarbete, 2013:64.

24. Laher F, Aremu AO, Van Staden J, and Finnie JF. Evaluating the effect of storage on the biological activity and chemical composition of three 
South African medicinal plants. South African Journal of Botany. 2013, Vol 88, pp 414-418.

25. Punniamurthy N, and Ramakrishnan N. Antimicrobial activity of ethnoveterinary herbal fresh preparation for mastitis. Kerala Veterinary Congress, Kerala, India, 2015, pp 398-401.

26. Andrews JM. Determination of minimum inhibitory concentrations. Journal of Antimicrobial Chemotherapy, 48 Suppl, 2001. S1 5-16.

27. Balakrishnan MN, and Punniamurthy $\mathrm{N}$. Ethno veterinary practices (EVP) as a new approach for management of cattle health without antimicrobial and other chemical veterinary drugs. EC Veterinary Science. 2021; 6.5. 